Check for updates

Cite this: RSC Adv., 2019, 9, 27665

Received 29th July 2019

Accepted 22nd August 2019

DOI: 10.1039/c9ra05859a

rsc.li/rsc-advances

\title{
Novel carbazole-based donor-isoindolo[2,1-a] benzimidazol-11-one acceptor polymers for ternary flash memory and light-emission $\uparrow$
}

\author{
Qian Zhang, ${ }^{\mathrm{a}}$ Chunpeng $\mathrm{Ai}^{\mathrm{b}}{ }^{\mathrm{b}}$ Dianzhong Wen, ${ }^{\mathrm{b}}$ Dongge $\mathrm{Ma},{ }^{\mathrm{c}}$ Cheng Wang, (D) a \\ Shuhong Wang (iD *a and Xuduo Bai (D) *a
}

\begin{abstract}
Three novel donor-acceptor polymers (PCzO, PCz2 and PCz4) based on 9-(9-heptadecanyl)-9Hcarbazole and isoindolo[2,1-a]benzimidazol-11-one with fluorine substituents $(0,2$ and 4$)$ on the acceptor unit were prepared by Suzuki polymerization. The synthesized polymers were studied by theoretical calculation, and optical and electrochemical characterizations to further investigate the performance of memory storage and light-emission. The memory devices of the three polymers all exhibited obvious ternary flash behavior with total ON/OFF state current ratio around $10^{4}$ and threshold voltages below $3.0 \mathrm{~V}$, with no other blending or doping. Bright emissions of an electroluminescence device based on PCZO was obtained at $556 \mathrm{~nm}$, the maximum brightness was $2006 \mathrm{~cd} \mathrm{~m}^{-2}$ with EQE of $0.21 \%$. The results suggested that polymers with the structure of carbazole-based donor as backbone and isoindolo[2,1-a]benzimidazol-11-one segment as acceptor could be excellent materials for memory storage and light-emitting applications with further investigation and could be used for further design of other new polymer systems.
\end{abstract}

\section{Introduction}

Resistive memory devices based on conductive polymers have attracted great interest ${ }^{1-4}$ on account of the advantages of polymeric materials such as the ease of manufacture by simply spin coating, with low consumption, large capacity for data storage, and feasibility of modification at the molecular level to achieve ideal electrical properties. With probes applied on two electrodes under a low voltage, the memristor would exhibit resistive write, read or erase states by distinguishing the digital 0 and 1 of bits. Apart from a typical binary memristor exhibiting two conductive states (ON and OFF states) that can store 2 bits, works on ternary or multilevel memory devices that can store more than 2 bits signals, which means more than one ON or OFF states would be observed during switching, are increasing as well. ${ }^{5-7}$ The electrical properties of a conductive polymer used as an active layer that is spin-coated and sandwiched between two electrodes play a key role to determine the resistive type of the memristor and affect the performance such as the threshold

${ }^{a}$ Key Laboratory of Functional Inorganic Material Chemistry, Ministry of Education, School of Chemistry Engineering and Materials Science, Heilongjiang University, Harbin, 150080, China

${ }^{b}$ Key Laboratories of Senior-Education for Electronic Engineering, Heilongjiang University, Harbin, 150080, China

'Institute of Polymer Materials, South China University of Technology, Guangzhou, 510640, China

$\dagger$ Electronic supplementary information (ESI) available. See DOI: $10.1039 / \mathrm{c} 9 \mathrm{ra} 05859 \mathrm{a}$ voltage and the ON/OFF current ratio of the device. There have been many works on polymer-based memristors for information storage that focused on blending polymers with nano materials or fullerene and its derivatives, and on doping polymers with ion or acid to obtain different types of memristors or to enhance the parameters of devices. For example, memory device based on PVK-CNT composite films behaved from insulator to WORM (write-once-read-many) to rewritable memory with blending different weight percentage of $\mathrm{CNT}{ }^{8}$ PFO-based memory device possessed an ON/OFF current ratio raising from $10^{1}$ to $10^{3}$ after blending with $1.6 \mathrm{wt} \%$ MWCNTs, ${ }^{9}$ a well-dispersed PCBM clusters within PCBM:PTPA hybrid films of ITO/PCBM:PTPA/Al memory device could exhibit from DRAM to WORM behaviour by controlling the PCBM content of $3 \%$ to $5 \mathrm{wt} \%{ }^{10}$ ion-doped poly(4-vinylpyridine) derivative-based memristor could be tuned from a binary to a ternary performance by increasing the content of carbazole moieties, ${ }^{5}$ and acid-doped polyazomethine (PA-TsOH)-based memory device with the configuration of $\mathrm{Pt} / \mathrm{PA}-\mathrm{TsOH} / \mathrm{Pt}$ demonstrated a multilevel storage capability. ${ }^{\mathbf{1 1}}$ Therefore, it's an important basic work to investigate polymeric material that possesses good memory performance itself, without additional blending or modification. Several types of polymers can be applied to fabricate memory devices in general, such as poly $(N$-vinyl carbazole)s (PVKs), ${ }^{\mathbf{8 , 1 2 - 1 4}}$ polyfluorenes (PFs), ${ }^{\mathbf{9}, 15,16}$ polytriphenylamines (PTPA), ${ }^{10,17,18}$ polyimides (PIs), ${ }^{19-21}$ polymers containing two or more segments mentioned above, ${ }^{22-26}$ and polymers containing metal complexes. ${ }^{27,28}$ Among these 
polymers, derivatives of PVK have exhibited excellent memory performances since the containing electron donating carbazole groups could facilitate hole transporting. Polymers containing carbazole groups possess two types of structures: carbazole as backbone and carbazole as a pendant linking to sidechain. The latter utilizes the tendency of neighbouring carbazole groups to form a face-to-face conformation that could facilitate charge carriers hopping through under an applied voltage, leading to a high-conductivity state, ${ }^{14,26,29,30}$ while the former fully utilizes the property of hole transporting, the advantage of rigid planar structure, and the ease of controlling the photoelectron performance at molecule level by introducing various acceptor directly to the backbone of the carbazole group. ${ }^{24,31}$ PIs have been widely used as acceptor because of the excellent thermal, chemical and electro properties and the feasible functionalization of molecule to tune these properties. The phthalimide segment of PI possesses strong ability of electron withdrawing and would achieve a conductive charge transfer (CT) state under applied electric field by linking with an electron donor to form a donor-acceptor (DA) system of a polymer, which could lead to a non-volatile behaviour of a memristor. Thus, the memory behaviours can be tuned by both electron donor and acceptor. ${ }^{32-35}$ In the light of above information, we designed and synthesized three DA polymers that using carbazole backbone as donor and choosing an isoindolo benzimidazole group substituted with different numbers of fluorine $(0,2,4)$ on the sidechain to tune the ability of electro transporting as acceptor, and studied the memory behaviours based on these polymers.

Since the fabrication of an asymmetric memory device is the same as the simplest configuration of a PLED device, which is substrate/bottom electrode/polymer/top electrode, ${ }^{36}$ and considering the PVKs have been widely used in optoelectronic field as an elector-donating and hole transporting group, ${ }^{37,38}$ we also prepared PLED devices using the designed polymers to find out the performance of light-emitting. In this study, we presented the synthesis of isoindolo[2,1-a]benzimidazol-11-one segments with different numbers of fluorine substitutions $(0$, $2,4)$ as acceptors introduced to 9-(9-heptadecanyl)-9H-carbazole backbone by Suzuki reaction to prepare and characterize three novel DA polymers (PCz0, PCz2 and PCz4), fabricated memory devices and PLEDs based on these polymers as functional layers and investigated the performances of these devices. As a result, the resistive switching memory devices of three polymers all exhibited ternary flash behaviour with total ON/OFF current ratio reached to about $10^{4}$ and threshold voltages below $3.0 \mathrm{~V}$. The PLED device based on PCz0 with the configuration of ITP/ PEDOT:PSS/polymer/TPBi/LiF/Al showed good emission at about $556 \mathrm{~nm}$ with maximum external quantum efficiency of $0.21 \%$ and maximum brightness of $2006 \mathrm{~cd} \mathrm{~m}^{-2}$. To the best of our knowledge, PCz0, PCz2 and PCz4 are few examples of promising copolymer containing carbazole group as backbone and phthalimide moiety as sidechain that processes obvious ternary flash memory behaviours with no other blending or doping. The results suggested that the designed polymers were worthy of further modification for higher performances of memory and PLED devices and of further research on designing other new polymer systems.

\section{Experimental}

\section{Materials and instruments}

3,6-Dibromobenzene-1,2-diamine purchased from YIMEI (Harbin, China) and phthalic anhydride purchased from J\&K (Beijing, China) were purified by sublimation $\left(10^{-2} \mathrm{mbar}\right)$ before use. All other chemicals purchased from TCI (Shanghai, China) and Aladdin (Shanghai, China) were used as received. All indium-tin oxide (ITO) substrates used for devices were well cleaned and treated by Photo Surface Processor (PL 16-110, replace lamp SUV110GS-36) before use.

FT-IR spectra were obtained from PerkinElmer Spectrum 100 Model FT-IR spectrometer. ${ }^{1} \mathrm{H}$-NMR spectra were obtained on Bruker AC-400 MHz NMR Spectrometer. GPC analysis was recorded by using a Malvern instrument connected with Viscotek-VE3580-RI-Detector and standard polystyrene samples as calibration, which were performed on a polymer/THF solution at a flow rate of $1 \mathrm{~mL} \min ^{-1}$ at $30^{\circ} \mathrm{C}$. Thermal gravimetric analyses (TGA) were taken under nitrogen atmosphere with $10{ }^{\circ} \mathrm{C} \min ^{-1}$ rate and were recorded on PerkinElmer Pyris 6 workstation. Photochemical measurements were obtained by using SHIMADZU UV-3600 and Jasco FP-6200 spectrophotometer. Cyclic voltammeter was measured by CHI-660E electrochemical workstation at a voltage scan rate of $50 \mathrm{mV} \mathrm{s}^{-1}$. Memory behaviour was carried out by Keithley 4200-SCS semiconductor workstation under applied voltage with step of $0.05 \mathrm{~V}$. Luminance properties of light-emitting device were recorded by using Keithley source measurement (Keithley 2400 and Keithley 2000) with a calibrated silicon photodiode. The electroluminescent spectra were obtained by using Spectra scan PR650 spectrophotometer.

\section{Synthesis of monomers and polymers}

The general synthesis procedure using $o$-phenylenediamine to prepare isoindolo[2,1-a]benzimidazol-11-one and its analogues have been fairly reported..$^{39,40} \mathrm{We}$ chose the typical conditions with heating under reflux in acetic acid and acetic anhydride successively to prepare isoindolo[2,1-a]-6,9-dibromobenzimidazol-11-one ("0f"), 2,3-difluoroisoindolo[2,1-a]-6,9-dibromobenzimidazol-11-one ("2f") and 1,2,3,4-tetrafluoroisoindolo[2,1-a]-6,9-dibromobenzimidazol11-one (" $4 \mathrm{f}$ "). FT-IR and ${ }^{1} \mathrm{H}$-NMR were used to verify the structural characterization of three monomers, which were described in ESI, $\uparrow$ along with the details of synthesis (see ESI S1 and S2†).

The general synthesis procedure of polymers was shown in Scheme 1. 9-(9-Heptadecanyl)-9H-carbazole-2,7-diboronic acid bis(pinacol) ester, monomer, $\mathrm{Pd}\left(\mathrm{PPh}_{3}\right)_{4}(3 \mathrm{~mol} \%$ based on total monomer) were dissolved in 6-8 $\mathrm{mL}$ of toluene and $1 \mathrm{~mL}$ of $2 \mathrm{M}$

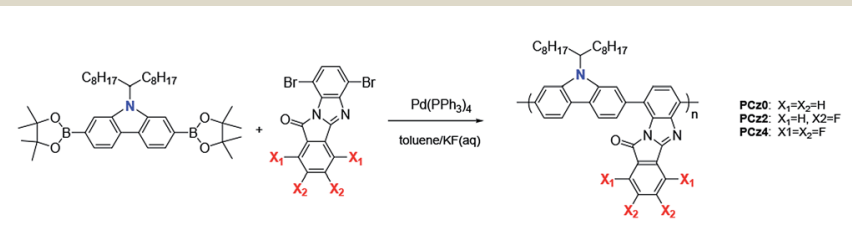

Scheme 1 Synthesis route of carbazole-fluorinated acceptor polymers PCzO, PCz2 and PCz4. 
$\mathrm{KF}$, degassed, backfilling with nitrogen and heated to reflux under nitrogen atmosphere for $72 \mathrm{~h}$. The resulting mixture was cooled to room temperature and extracted from toluene 3 times, concentrated to a small amount and re-precipitated from $180 \mathrm{~mL}$ of methanol $/ \mathrm{H}_{2} \mathrm{O}(8: 1, \mathrm{v} / \mathrm{v})$. The precipitate was collected, washed with acetone for $48 \mathrm{~h}$ by Soxhlet extractor method, and finally dried under vacuum.

\section{Poly[2,7-9-(9-heptadecanyl)-9H-carbazole-co-benzo[4,5] imidazo[2,1-a]isoindol-11-one] (PCz0)}

$0.197 \mathrm{~g}$ (0.3 mmol) of 9-(9-heptadecanyl)-9H-carbazole-2,7diboronic acid bis(pinacol) ester, $0.113 \mathrm{~g}(0.3 \mathrm{mmol})$ of 6,9dibromobenzo[4,5]imidazo[2,1- $a$ ]isoindol-11-one (0f), $12 \mathrm{mg}$ of $\mathrm{Pd}\left(\mathrm{PPh}_{3}\right)_{4}$ and $8 \mathrm{~mL}$ of toluene were used to afford $0.116 \mathrm{~g}$ of purified yellow green solid PCz0 (62.0\%). ${ }^{1} \mathrm{H}-\mathrm{NMR}(400 \mathrm{MHz}$, $\left.\mathrm{CDCl}_{3}\right): \delta(\mathrm{ppm})$ 0.84-0.92 (m, 6H, $\left.\mathrm{CH}_{3}\right), 1.15-1.54(\mathrm{~m}, 22 \mathrm{H}$, $\mathrm{CH}_{2}$ ), 1.91-2.34 (m, 6H, $\mathrm{CH}_{2}$ ), 3.60-3.72 (m, 1H, CH), 7.67-8.66 (m, 12H, Ar-H).

\section{Poly[2,7-9-(9-heptadecanyl)-9H-carbazole-co-2,3- difluorobenzo[4,5]imidazo[2,1-a]isoindol-11-one] (PCz2)}

$0.197 \mathrm{~g}$ (0.3 mmol) of 9-(9-heptadecanyl)-9H-carbazole-2,7diboronic acid bis(pinacol) ester, $0.124 \mathrm{~g}(0.3 \mathrm{mmol})$ of 2,3difluoro-6,9-dibromobenzo[4,5]imidazo[2,1-a]isoindol-11-one

(2f), $17 \mathrm{mg}$ of $\mathrm{Pd}\left(\mathrm{PPh}_{3}\right)_{4}$ and $6 \mathrm{~mL}$ of toluene were used to afford $0.132 \mathrm{~g}$ of purified yellow green solid PCz2 (66.7\%). ${ }^{1} \mathrm{H}-\mathrm{NMR}$ (400 $\left.\mathrm{MHz}, \mathrm{CDCl}_{3}\right): \delta(\mathrm{ppm})$ 0.74-0.91 (m, 6H, $\left.\mathrm{CH}_{3}\right), 1.15-1.46$ (m, 22H, $\mathrm{CH}_{2}$ ), 1.93-2.37 (m, 6H, $\left.\mathrm{CH}_{2}\right), 3.59-3.68(\mathrm{~m}, 1 \mathrm{H}, \mathrm{CH})$, 7.52-8.19 (m, 10H, Ar-H).

Poly[2,7-9-(9-heptadecanyl)-9H-carbazole-co-1,2,3,4tetrafluoro-benzo[4,5]imidazo[2,1-a]isoindol-11-one] (PCz4)

$0.197 \mathrm{~g}$ (0.3 mmol) of 9-(9-heptadecanyl)-9H-carbazole-2,7diboronic acid bis(pinacol) ester, $0.135 \mathrm{~g}(0.3 \mathrm{mmol})$ of 1,2,3,4-tetrafluoro-6,9-dibromobenzo[4,5]imidazo[2,1- $a]$

isoindol-11-one (4f), $12 \mathrm{mg}$ of $\mathrm{Pd}\left(\mathrm{PPh}_{3}\right)_{4}$ and $6 \mathrm{~mL}$ of toluene were used to afford $0.107 \mathrm{~g}$ of purified orange-yellow solid $\mathrm{PCz} 4$ (51.2\%). ${ }^{1} \mathrm{H}-\mathrm{NMR}\left(400 \mathrm{MHz}, \mathrm{CDCl}_{3}\right): \delta(\mathrm{ppm})$ 0.84-0.87 (m, 6H, $\mathrm{CH}_{3}$ ), 1.16-1.45 (m, 22H, $\mathrm{CH}_{2}$ ), 1.99-2.39 (m, 6H, $\left.\mathrm{CH}_{2}\right), 3.57-$ 3.67 (m, 1H, CH), 7.37-8.33 (m, 8H, Ar-H).

\section{Devices preparation}

The memory devices were fabricated with the configuration of ITO/polymer/Al. Polymers in $10 \mathrm{mg} \mathrm{mL}^{-1}$ dichlorobenzene solution (filtrated by $0.45 \mu \mathrm{m}$ PTFE filter after dissolved) were spin coated on top of ITO substrates at $500 \mathrm{rpm}$ for $1 \mathrm{~min}$ and dried at $80{ }^{\circ} \mathrm{C}$ under nitrogen. The samples were transferred to a thermal evaporation chamber with a shadow mask for $\mathrm{Al}$ deposition under high vacuum of $2 \times 10^{-6} \mathrm{mbar}$. The diameter of the top Al electrode of memory device is $200 \mu \mathrm{m}$. The PLED devices were fabricated with the configuration of ITO/ PEDOT:PSS/polymer/TPBi/LiF/Al. PEDOT:PSS was spin-coated on top of the ITO substrates at $1000 \mathrm{rpm}$ for $1 \mathrm{~min}$ and dried at $80{ }^{\circ} \mathrm{C}$ for $1 \mathrm{~h}$. Polymers solved in THF as $10 \mathrm{mg} \mathrm{mL}^{-1}$ solutions (filtrated by $0.45 \mu \mathrm{m}$ PTFE filter after dissolved) were then spin-coated above the PEDOT:PSS layer at $1500 \mathrm{rpm}$ for $1 \mathrm{~min}$. TPBi, LiF and Al were deposited under high vacuum of $1 \times 10^{-6}$ mbar with a shadow mask to give the device an active area of 16 $\mathrm{mm}^{2}$. Preparation steps after spin coating PEDOT:PSS were carried out in two interconnected $\mathrm{N}_{2}$-filled glove boxes $\left(\left[\mathrm{O}_{2}\right]\right.$ $\left.<3 \mathrm{ppm},\left[\mathrm{H}_{2} \mathrm{O}\right]<0.5 \mathrm{ppm}\right)$.

\section{Results and discussion}

\section{Characterization of PCz0, PCz2 and PCz4}

The peaks of ${ }^{1} \mathrm{H}-\mathrm{NMR}$ resonated in the region of $0.74-2.39 \mathrm{ppm}$, 3.57-3.72 ppm and 7.37-8.66 ppm were assigned to the aliphatic $\left(-\mathrm{CH}_{3},-\mathrm{CH}_{2}\right)$, aliphatic $(-\mathrm{CH})$ and aromatic protons, respectively. In the case of FT-IR spectrum, polymers exhibited similar characteristic peaks due to their similar structure. Peaks around 2853-2926 $\mathrm{cm}^{-1}$ was typical strong $\mathrm{C}-\mathrm{H}$ stretching for octyl chains, peaks around $1213 \mathrm{~cm}^{-1}$ and $1345 \mathrm{~cm}^{-1}$ were alkyl and aryl $\mathrm{C}-\mathrm{N}$, and it's noticeable that stretching vibrations of carbonyl groups in condensed cyclic $\gamma$-lactams would move to about $1770 \mathrm{~cm}^{-1}$ in their IR spectra. ${ }^{40}$ The $\mathrm{C}-\mathrm{F}$ bonds don't usually possess a constant vibrational frequency nor do they always have unique absorption band features, which may lead to the difficulty of locating and recognizing their absorption. ${ }^{\mathbf{4 1}}$ Since the C-F stretching could exhibit two or more bands of polyfluorinated aliphatic hydrocarbons with a broad range ${ }^{41}$ of 1400-1000 $\mathrm{cm}^{-1}$, and compared with the non-fluorinated monomer of, the aromatic $\mathrm{C}-\mathrm{F}$ stretching bands of fluorinated monomer $2 \mathrm{f}$ and $4 \mathrm{f}$ were assumed to be $\left(1471 \mathrm{~cm}^{-1}\right.$, $1486 \mathrm{~cm}^{-1}$ ) and $\left(1492 \mathrm{~cm}^{-1}, 1519 \mathrm{~cm}^{-1}\right)$, respectively. The $\mathrm{C}-\mathrm{F}$ vibration of polymer $\mathrm{PCz} 2$ was hard to recognize, and in terms of $\mathrm{PCz} 4$, the $\mathrm{C}-\mathrm{F}$ stretching band could be located around $1508 \mathrm{~cm}^{-1}$ (see ESI S3 and $\mathrm{S} 4 \dagger$ ). Prepared polymers of PCz0, PCz2 and PCz4 possessed the $M_{\mathrm{n}}$ of 12916 with the polydispersity indices (PDI) of 1.26, 13289 with PDI of 1.20 and 12826 with PDI of 1.26 , respectively, which could well meet the requirements of spin-coating thin-layers in fabricating process of device.

\section{Theoretical quantum calculation of polymers}

The molecular simulation based on the optimized repeat unit of PCz0, PCz2 and PCz4 were carried out by hybrid density functional theory B3LYP/6-31G basis set of Gaussian 09 program in order to get some insight into the properties of the polymers. As shown in Table 1, the HOMOs were nearly all located on the donor moieties, while the LUMOs were located on the acceptor moieties. The dihedral angles between donor and acceptor units of model polymers were $46.0^{\circ}, 49.5^{\circ}$ and $46.2^{\circ}$, respectively, which indicated there may be conformational distortion and the intramolecular charge transfer in ground state may not be effective. ${ }^{42}$ As the numbers of fluorine introducing to the acceptor increased, the positive region (blue) of the electrostatic potential surfaces (ESP) was more dispersed in doublyfluorinated model of $\mathrm{PCz} 2$ while more centralized in fourfluorinated model of PCz4. According to the definition of ESP, positive charges approaching molecule would favor the negative region, the more electron-rich sites, and vice versa. ${ }^{43}$ Thus, once 
Table 1 Calculated molecular orbitals and energy levels and ESP of the basic units of $\mathrm{PCzO}, \mathrm{PCz} 2$ and $\mathrm{PC} 44$

\begin{tabular}{|c|c|c|c|}
\hline & HOMO & LUMO & Electrostatic potential surfaces (ESP) \\
\hline PCz0 & & $\begin{array}{ll}3 \\
3\end{array}$ & \\
\hline PCz2 & & 3 & \\
\hline PCz4 & & & \\
\hline
\end{tabular}

under applied electric field, holes and electrons injected from electrodes could be trapped/detrapped by the negative and positive regions of a polymer respectively, and consequently affect the balance of electron accumulation and transportation. The dipole moment calculation results of three models were 3.50 of PCz0, 1.69 of PCz2 and 1.87 Debye of PCz4, which were assumed to be stable enough to sustain the CT state that formed under electric field and memristors based on these polymers might exhibited WORM or flash memory behaviors. ${ }^{3}$ These results also suggested the optical and electro performance of three polymers may not scale with the number of fluorine substitutions.

\section{Thermal, optical properties and electrochemical characteristics of polymers}

The TGA (see ESI S5†) of PCz0, PCz2 and PCz4 showed good thermal stability, of which the onset decomposition temperatures $\left(T_{\mathrm{d}}\right)$ were $331{ }^{\circ} \mathrm{C}, 362{ }^{\circ} \mathrm{C}$ and $330{ }^{\circ} \mathrm{C}$, respectively, which met requirement well with the stability of polymers. Fig. 1 showed the absorption and emission of PCz0, PCz2 and PCz4 in thin films. The absorption of three polymers possessed two main regions, $\pi-\pi *$ transition peaks around $310 \mathrm{~nm}$ that might attribute to the donor unit and $\pi-\pi^{*}$ transition that might

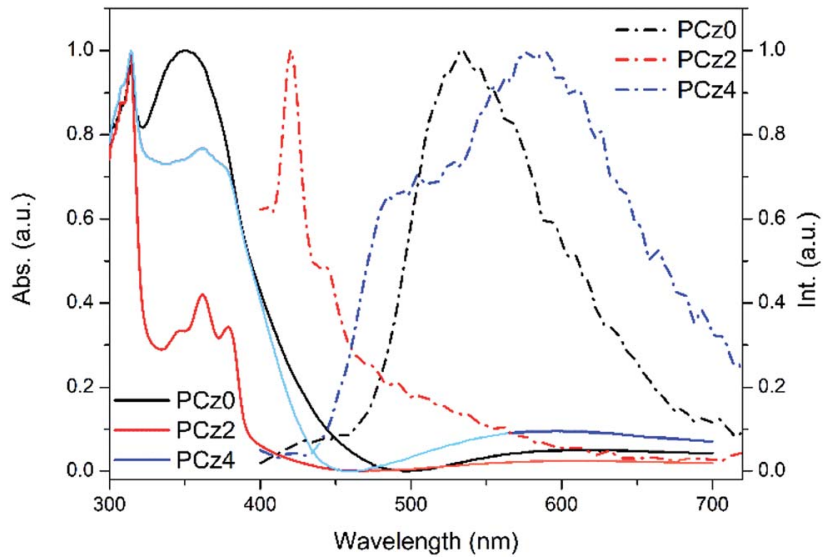

Fig. 1 Normalized UV-vis absorption (solid) and PL spectra (dash) of $\mathrm{PCz} 0, \mathrm{PCz} 2$ and $\mathrm{PCz} 4$ in thin films. cause by the acceptor unit. No obvious absorption above $400 \mathrm{~nm}$ were observed, which indicated there may be conformational distortion that restricted the charge transfer between donor and acceptor segment. ${ }^{42}$ The emission spectra of PCz2 exhibited stocks shift about $58 \mathrm{~nm}$, while more than $100 \mathrm{~nm}$ of stocks shift were found in the case of $\mathrm{PCz} 0$ and $\mathrm{PCz} 4$, which exhibited new dominated green emission while blue emission band were weakened. This may attribute to less twist angle in main chain of the two polymers that may result in more efficient excitation energy transfer that facilitated by intra- and interchain interaction. ${ }^{44,45}$ The optical properties of three polymers were summarized in Table 2.

The highest molecular orbital (HOMO) energy level was calculated from the formula $E_{\mathrm{HOMO}}=-\left(E_{\mathrm{Ox}}^{\text {onset }} v s . \mathrm{Ag} / \mathrm{AgCl}+\right.$ 4.4) eV, and the lowest unoccupied molecular orbital (LUMO) energy level was estimated from $E_{\mathrm{LUMO}}=E_{\mathrm{HOMO}}+E_{\mathrm{g}}^{\mathrm{opt}}$, on the base of which the HOMO and LUMO level were obtained as listed in Table 2. The HOMO levels of PCz0, PCz2 and PCz4 were quite approximated, but LUMO levels were more affected by the presence of fluorine, and the impact affected more on doublyfluorinated polymer PCz2 than the four-fluorinated polymer PCz4. The results also suggested that the strong electronwithdrawing nature of fluorine substituents were not the single factor that affect the optical and electrochemical properties of polymers and further behaviour in devices (Fig. 2).

\section{Characteristics of memory device}

The memory devices were fabricated with the simple structure of ITO/polymer/Al configuration (Fig. 3), along with the scanning electron microscopic images (SEM) of polymeric layer. With the thickness around $59 \mathrm{~nm}$, the surface of polymer was uniformed. The memory behaviour was investigated by currentvoltage $(I / V)$ characteristics and retention behaviours, which the value of current was replotted on a log scale (Fig. 4). As device based on PCz0 (Fig. 4a) for example, the first sweep under the voltage ranged from 0 to $-6 \mathrm{~V}$ exhibited an OFF-to-ON transition which was defined as a "writing" process. With the increase applied bias during the first sweep, the current showed a sudden jump at $-1.1 \mathrm{~V}$, which revealed device switching from a low conductive state (OFF state) to a high conductive state (ON-1 state), the current kept increasing as the voltage rising, and jumped to a high conductive state (ON-2 state) under $-2 \mathrm{~V}$. At this first sweep, the current jumped from about $10^{-5} \mathrm{~A}$ to $10^{-2} \mathrm{~A}$ (ON-1 state) and finally to $10^{-1} \mathrm{~A}$ (ON-2 state), which indicated the total ON/OFF ratio reached to $10^{4}$. The device remained at the ON state during the whole second sweep, which was considered as a "reading" behaviour of the device. With the applied bias reversed, the device exhibited an ON-to-OFF transition which was defined as an "erasing" process. The device remained at ON state until the applied bias increased to $2.2 \mathrm{~V}$, under which the device switched to OFF state. During the last sweep, the device remained at OFF state as a "re-reading" behaviour. The intermediate resistance state like ON-1 under negative bias was not observed during the sweep of positive bias, which probably due to the residual conductive filaments formed during the first and second sweep through which the 
Table 2 Optical and electrochemical characteristics of PCzO, PCz2 and PCz4

\begin{tabular}{llllll}
\hline Polymer & Abs $\lambda_{\max }{ }^{a}(\mathrm{~nm})$ & Abs $\lambda_{\text {onset }}(\mathrm{nm})$ & $\mathrm{PL}_{\max }{ }^{b}(\mathrm{~nm})$ & $E_{\mathrm{g}}^{\mathrm{optc}}(\mathrm{eV})$ & $E_{\mathrm{HOMO}}(\mathrm{eV})$ \\
\hline PCz0 & 350 & 458 & 534 & 2.71 & -5.43 \\
PCz2 & 362 & 396 & 420 & 3.13 & -2.72 \\
PCz4 & $360-363$ & 443 & 504,578 & 2.80 & -2.33 \\
\end{tabular}

${ }^{a}$ Only the lowest energy maxima was shown. ${ }^{b}$ Excited at $370 \mathrm{~nm} \cdot{ }^{c}$ Estimated from the onset of the absorption of the polymer in thin film as $E_{\mathrm{g}}^{\text {opt }}=$ $1240 / \lambda_{\text {onset }}{ }^{d}$ Calculated as $E_{\mathrm{LUMO}}=E_{\mathrm{HOMO}}+E_{\mathrm{g}}^{\mathrm{opt}}$.

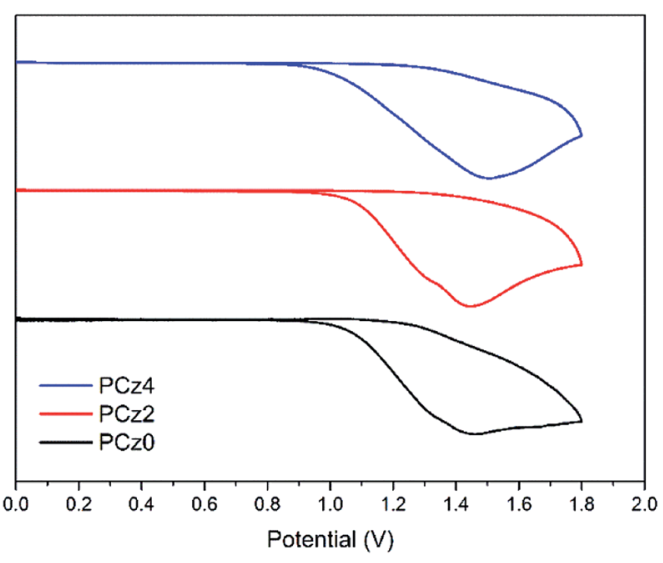

Fig. 2 Cyclic voltammetry of polymer PCzO, PCz2 and PCz4.

current could flow as a channel, thus the intermediated state may not sustain under the lower positive bias. ${ }^{7,46,47}$ The rewritable and non-volatile four stepped circle revealed a ternary flash type memory device with an ON/OFF current ratio greater than $10^{4}$.

The devices based on PCz2 and PCz4 exhibited similar flash behaviour (Fig. $4 \mathrm{~b}$ and c). In the second voltage scan from $0 \mathrm{~V}$ to $-6 \mathrm{~V}$, the current of two devices jumped to ON-1 state under $-1.3 \mathrm{~V}$ and $-0.9 \mathrm{~V}$, and subsequently to $\mathrm{ON}-2$ state under $-3.6 \mathrm{~V}$ and $-1.5 \mathrm{~V}$, respectively. While in the third voltage scan from 0 to $6 \mathrm{~V}$, the current of two devices sharp dropped to OFF state under $3.8 \mathrm{~V}$ and $4.0 \mathrm{~V}$, respectively. Compared to the device based on PCz0, devices with PCz2 and PCz4 possessed much higher ON-1/ON-2 ratio that could reach to around $10^{4}$ (see ESI S6 $\dagger$ ). These two devices exhibited ternary flash type as well. Fig. $4 \mathrm{~d}-\mathrm{f}$ showed the retention behaviours of OFF, ON-1 and $\mathrm{ON}-2$ states of memory devices. Under the voltage of $-0.5 \mathrm{~V}$, no obvious degradation was observed for at least $5000 \mathrm{~s}$.

Among three polymers, the switching-on voltage of double fluorinated polymer was the highest, which may due to the highest band gap $\left(E_{\mathrm{g}}\right)$ of PCz2 $(3.13 \mathrm{eV})$, while the $E_{\mathrm{g}}$ of PCz0 and $\mathrm{PCz} 4$ were close $(2.71 \mathrm{eV}$ and $2.80 \mathrm{eV})$, leading to a similar onvoltage of the two. As discussed in the theoretical quantum calculation section, the dipole moment played an important role to sustain the CT state formed under electric field during the working of a flash type memristor, ${ }^{3}$ thus the PCz0 exhibited the highest dipole moment of $3.50 \mathrm{D}$ resulting in a more balanced and stable curves. Although memory device of PCz2 exhibited higher on-voltage and less stable $I / V$ characteristics,
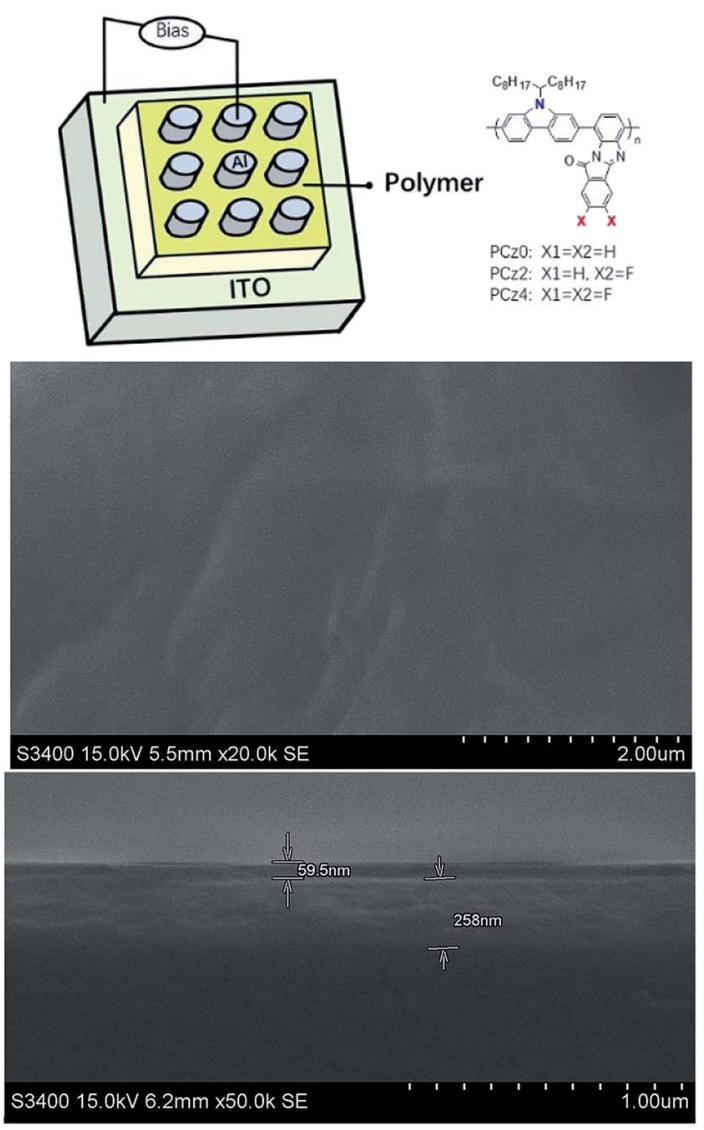

Fig. 3 Illustration and SEM images of the ITO/polymer/Al memory device.

the ON/OFF ratio was the largest (see ESI S6†). These results indicated that the tunability of resistivity in molecule level was feasible and the improvement of the memory performance may not scale with the increasing of fluorine substituents.

One of the most widely used mechanisms of resistive memory devices based on donor-acceptor polymer is the charge transfer effect. When the bias reached the threshold voltage (the switching-on voltage) of the memory device, partial of charges at the HOMO level transited to various LUMO levels with the highest probability to reach the excited state, leading to a formation of high conductivity path within polymers. ${ }^{48,49}$ When the applied voltage was removed, the charge transfer state could be sustained by the formation of dipole moment in polymer, which resulting in a non-volatile memory behavior. ${ }^{50}$ 

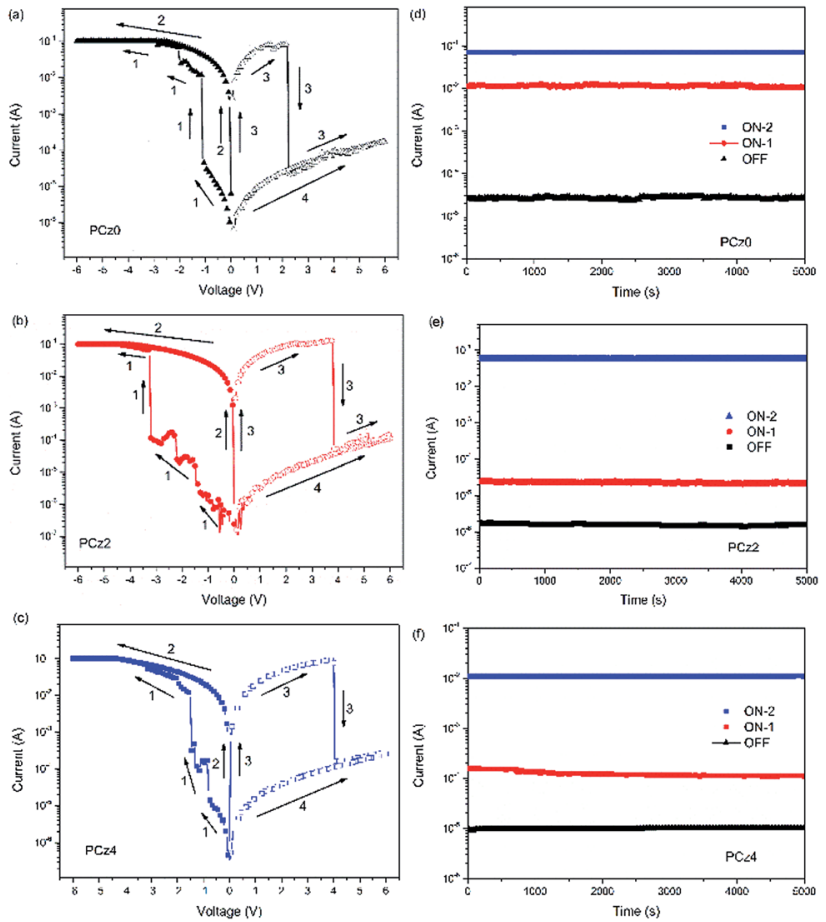

Fig. 4 Current-voltage $(I-V)$ characteristics and retention behaviours under $-0.5 \mathrm{~V}$ of $\mathrm{PCzO}$ ( $a$ and d), PCz2 (b and e) and PCz4 (c and f).

In the present PCz0 as example (Fig. 5), as the energy levels of HOMO and LUMO3 of PCz0 were overlapped, charges with enough energy transiting from HOMO to LUMO3 within the donor unit to form an excited state was a plausible process and the charges of LUMO3 of donor could transfer to LUMO2 and LUMO of acceptor afterwards. Besides, the charges from HOMO of donor could also transfer directly to the LUMO2 and LUMO level of acceptor but with different probabilities. The multiple transition processes of charges would promote intermolecular

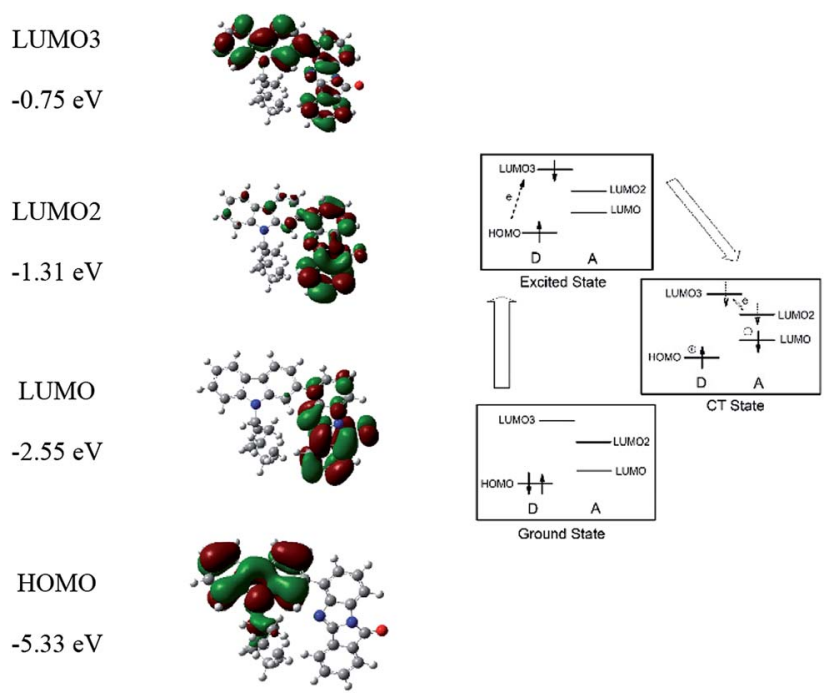

Fig. 5 Illustration of transitions induced by the electric field based on optimized repeated unit of $\mathrm{PCzO}$. charge transfer to form a conductive complex, thus, high conductive states of PCz0 were reached, leading to sharp current increases and sustained ON states of the memory device. During the reverse sweep, the holes were injected from ITO electrode and migrated throughout the backbone of PCz0, thus the applied voltage was required to supply the energy to dissociated the charge transfer complex and turn the device to initial OFF state. PCz2 and PCz4 exhibited similar transition patterns induced by the electric fields (see ESI S7 7 ).

Another way of understanding the mechanism of the memristor is the SCLC (space charge limited conduction) model for switching behaviour. Take memory device based on PCz0 (Fig. 6a) for example, at the beginning of charge injection from electrodes, the $I-V$ curve in log-log scale of OFF state exhibited linear (slope closed to 1) under lower voltage, which indicated
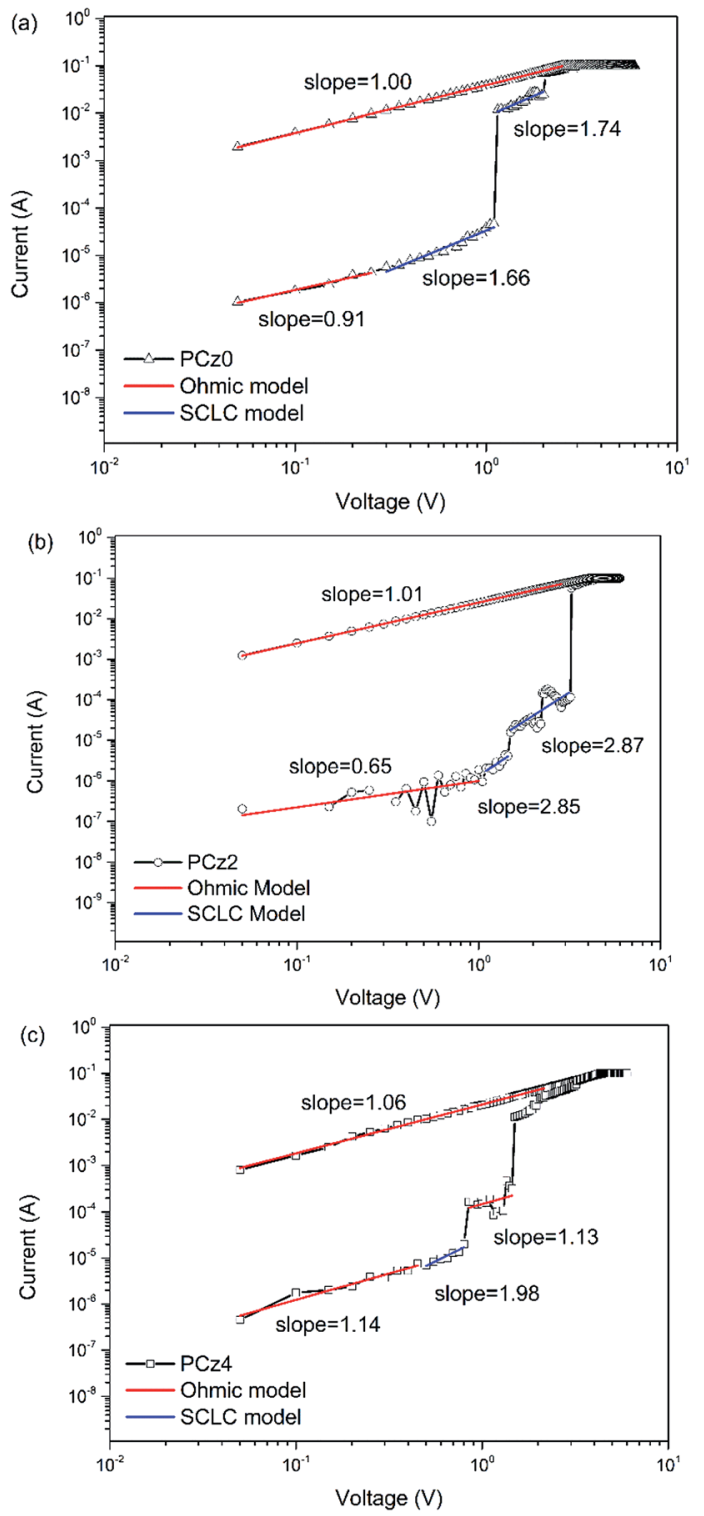

Fig. 6 Experimental $I-V$ curves (black line and symbol) in log-log pattern and fitting slopes (coloured lines) of memory device based on (a) PCzO, (b) PCz2 and (c) PCz4. 
the working mechanism followed ohmic conduction model as $J$ $\propto \mathrm{V}$ and the device was at a high resistance state. ${ }^{51,52}$ As applied voltage increasing, the current raised more slowly and the slope of curve could be nearly 2 (1.66 in this case), as the behavior of device was controlled by trapped carriers in this process as discussed above and the pattern was SCLC. ${ }^{53}$ After a sudden jump of current at on-voltage, the device switched from OFF state to $\mathrm{ON}-1$ state and then to the $\mathrm{ON}-2$ state, and the memory behavior was finally dominated by the ohmic conduction again. Memory devices of PCz2 and PCz4 exhibited similar $I-V$ curve region (Fig. 6b and c) and fitted the SCLC model as well.

\section{Characteristics of electroluminescence device}

On the basis of memory device, active layers of PEDOT:PSS and TPBi was inserted between electrode and polymer layer to configurated a PLED device with the structure of ITO/ PEDOT:PSS/polymer/TPBi/LiF/Al to find out the utility of polymer for electroluminescence (EL) of PCz0, PCz2 and PCz4. PEDOT:PSS was used to facilitate the hole injection and TPBi was used as an electron transporting and hole blocking layer. LiF deposited on $\mathrm{Al}$ electrode was used for lowering the barrier between work function of cathode and the LUMO energy level of active layers to enhance electron injection. Work function and energy levels were illustrated as Fig. 7.

The $V-I-B$ characteristics of PLED devices were shown in Fig. 8 and Table 3. Under applied bias, holes injected from ITO and electron injected from cathode recombined in the polymer layer, thus the higher the current density the lower the on voltage. The Homo energy levels of $\mathrm{PCz} 0, \mathrm{PCz} 2$ and $\mathrm{PCz} 4$ were similar, thus the LUMOs played an important role in the term of balance of carrier transport. As studied in the electro electrochemical characteristics section, PCz2 possessed the highest LUMO among the three polymers, which indicated the biggest barrier between polymer layer and electrode and led to the weakest behave of a PLED device. Compared to device of PCz4, PCz0 well matched the energy level of layers, resulting in the highest properties with maximum brightness of $2006 \mathrm{~cd} \mathrm{~m}^{-2}$

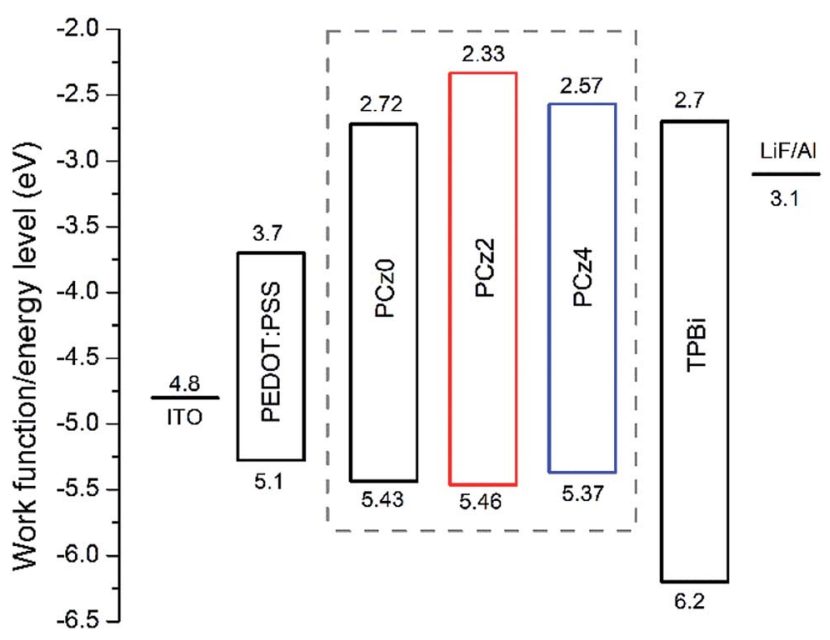

Fig. 7 Illustration of energy levels of all layers of PLED device.
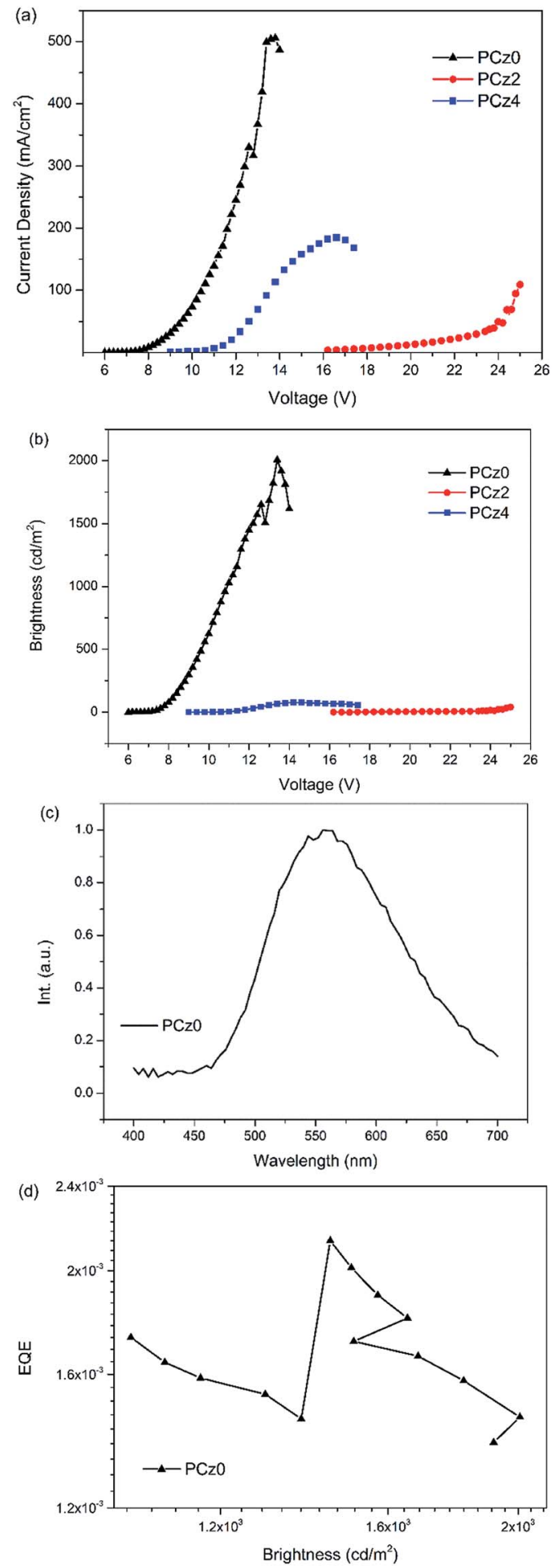

Fig. 8 (a) Current density and (b) brightness of ITO/PEDOT:PSS/ polymer/TPBi/LiF device based on PCzO, PCz2 and PCz4, (c) EL spectrum and (d) EQE of PLED device based on PCzO.

under $13.6 \mathrm{~V}$, almost 30-50-fold increase compared with the other two devices. The electroluminescent spectra of PCz0 exhibited maximum emission around $556 \mathrm{~nm}$ with the external quantum efficiency based on EL of $0.21 \%$, the other two were too weak to be detected. 
Table 3 Electroluminescence characteristics of PLEDs based on PCzO, PCz2 and PCz4 with active area of $0.16 \mathrm{~cm}^{2}$

\begin{tabular}{|c|c|c|c|c|c|c|c|}
\hline Polymer & $\lambda_{\max }^{\mathrm{EL}}(\mathrm{nm})$ & $\begin{array}{l}\text { On voltage } \\
\text { (V) }\end{array}$ & $\begin{array}{l}\text { Current density } \\
\left(\mathrm{mA} \mathrm{cm}^{-2}\right)\end{array}$ & $\begin{array}{l}\text { Brightness } \\
\left(\mathrm{cd} \mathrm{m}^{-2}\right)\end{array}$ & $\begin{array}{l}\text { Current efficiency }{ }^{\max } \\
\left(\mathrm{cd} \mathrm{A}^{-1}\right)\end{array}$ & Power efficiency $^{\max }(\mathrm{lm} / \mathrm{W})$ & $\mathrm{EQE}^{\max }(\%)$ \\
\hline PCz0 & 556 & 6.4 & 505.6 & 2006 & 0.96 & 0.38 & 0.21 \\
\hline PCz4 & $-^{a}$ & 10 & 184.8 & 77 & 0.10 & 0.03 & $-{ }^{a}$ \\
\hline
\end{tabular}

${ }^{a}$ Too weak to be detected.

\section{Conclusions}

Three carbazole-based donor-isoindolo[2,1-a]benzimidazol-11one acceptor polymers with comparable number of fluorine substitutions (0,2 and 4) substituted on acceptors were synthesized and studied. Introducing fluorine to acceptor unit could noticeably change the optical and electrochemical properties of polymers and played a certain role on memory and light-emitting behaviours, but the impact may not scale with the number of fluorine substitution increasing, at least in our studied series (PCz0, PCz2 and $\mathrm{PCz} 4$ ). Three polymer-based memristors all exhibited obvious ternary flash memory behaviours with low threshold voltage under $3.0 \mathrm{~V}$ and total ON/OFF state current ratio that could reach to $10^{4}$, with no additional blending or doping. Bright emissions of electroluminescence device based on PCz0 was obtained at $556 \mathrm{~nm}$, the maximum brightness was $2006 \mathrm{~cd} \mathrm{~m}^{-2}$ with EQE of $0.21 \%$. Considered that fluorine was substituted to the sidechain of acceptor unit other than directly into the backbone of polymer, the performance of devices might be affected by various contribution instead of the sole effect of strong electron-withdrawing nature of fluorine. The results indicated that the memory behaviours and electroluminescent performance could be tuned by modifying donor and acceptor segments, and these studied polymers could be considered for further use for other polymer systems and for further modification for the improvement of both memory storage and display applications.

\section{Conflicts of interest}

There are no conflicts to declare.

\section{Acknowledgements}

This research was supported by the National Natural Science Foundation of China (Grant no. 21372067, no. 51527804), the Doctoral Fund of Ministry of Education of China (Grant no. 20132301110001) and the Natural Science Foundation of Heilongjiang Province (Grant no. B2017010).

\section{Notes and references}

1 B. Cho, S. Song, Y. Ji, T.-W. Kim and T. Lee, Adv. Funct. Mater., 2011, 21, 2806.

2 T. Kurosawa, T. Higashihara and M. Ueda, Polym. Chem., 2013, 4, 16.
3 W. Lin, S. Liu, T. Gong, Q. Zhao and W. Huang, Adv. Mater., 2014, 26, 570.

4 Y. Chen, G. Liu, C. Wang, W. Zhang, R.-W. Li and L. Wang, Mater. Horiz., 2014, 1, 489.

5 D. He, H. Zhang, H. Liu, H. Liu, H. Li and J. Lu, J. Mater. Chem. C, 2013, 1, 7883.

6 P.-Y. Gu, F. Zhou, J. K. Gao, G. Li, C. Y. Wang, Q.-F. Xu, Q. C. Zhang and J.-M. Lu, J. Am. Chem. Soc., 2013, 135, 14086.

7 Y. Sun, J. Lu, C. Ai, D. Wen and X. Bai, Org. Electron., 2016, 32, 7.

8 G. Liu, Q.-D. Ling, E. Y. H. Teo, C.-X. Zhu, D. S.-H. Chan, K.-G. Neoh and E.-T. Kang, ACS Nano, 2009, 3, 1929.

9 Y. Xin, X. Zhao, H. Zhang, S. Wang, C. Wang, D. Ma and P. Yan, Org. Electron., 2019, 74, 110.

10 C.-J. Chen, Y.-C. Hu and G.-S. Liou, Chem. Commun., 2013, 49, 2804.

11 B. Hu, X. Zhu, X. Chen, L. Pan, S. Peng, Y. Wu, J. Shang, G. Liu, Q. Yan and R.-W. Li, J. Am. Chem. Soc., 2012, 134, 17408.

12 B. Zhang, G. Liu, Y. Chen, C. Wang, K.-G. Neoh, T. Bai and E.-T. Kang, ChemPlusChem, 2012, 77, 74.

13 B. Zhang, L. Liu, L. Wang, B. Liu, X. Tian and Y. Chen, Carbon, 2018, 134, 500.

14 X. Tian, Y. Cao, B. Zhang, S. Huang and Y. Chen, Eur. Polym. J., 2018, 106, 196.

15 T.-W. Kim, S.-H. Oh, H. Choi, G. Wang, H. Hwang, D.-Y. Kim and T. Lee, Appl. Phys. Lett., 2008, 92, 253308.

16 H.-C. Wu, A.-D. Yu, W.-Y. Lee, C.-L. Liu and W.-C. Chen, Chem. Commun., 2012, 48, 9135.

17 Y.-K. Fang, C.-L. Liu, G.-Y. Yang, P.-C. Chen and W.-C. Chen, Macromolecules, 2011, 44, 2604.

18 W. Zhang, C. Wang, G. Liu, J. Wang, Y. Chen and R.-W. Li, Chem. Commun., 2014, 50, 11496.

19 N. H. You, C. C. Chueh, C. L. Liu, M. Ueda and W. C. Chen, Macromolecules, 2009, 42, 4456.

20 S. Park, K. Kim, D. M. Kim, W. Kwon, J. Choi and M. Ree, ACS Appl. Mater. Interfaces, 2011, 3, 765.

21 S. H. Liu, W. L. Yang, C. C. Wu, T. S. Chao, M. R. Ye, Y. Y. Su, P. Y. Wang and M. J. Tsai, IEEE Electron Device Lett., 2013, 34, 123.

22 T. J. Lee, Y.-G. Ko, H.-J. Yen, K. Kim, D. M. Kim, W. Kwon, S. G. Hahm, G.-S. Liou and M. Ree, Polym. Chem., 2012, 3, 1276.

23 T.-T. Huang, C.-L. Tsai, S.-H. Hsiao and G.-S. Liou, RSC Adv., 2016, 6, 28815. 
24 J. Zhao, L. Peng, Y.-L. Zhu, Y.-J. Song, L.-J. Wang and Y.-Z. Shen, Polymer, 2016, 91, 118.

25 Q. Yang, X. Jiang, Y. Xin, X. Zhao, J. Huang, S. Wang, R. Zheng, D. Ma and C. Wang, RSC Adv., 2017, 7, 54431.

26 Y. Yang, H. Lu, J. Liu and Y. Shen, Eur. Polym. J., 2018, 108, 10.

27 Q.-D. Ling, W. Wang, Y. Song, C.-X. Zhu, D. S.-H. Chan, E.-T. Kang and K.-G. Neoh, J. Phys. Chem. B, 2006, 110, 23995.

28 S. J. Liu, P. Wang, Q. Zhao, H. Y. Yang, J. Wong, H. B. Sun, X. C. Dong, W. P. Lin and W. Huang, Adv. Mater., 2012, 24, 2901.

29 N. Jia, G. Tian, S. Qi, X. Wang and D. Wu, Eur. Polym. J., 2017, 95, 186.

30 T. M. McFarlane, B. Zdyrko, Y. Bandera, D. Worley, O. Klep, M. Jurca, C. Tonkin, S. H. Foulger, J. Vilcakova, P. Saha and J. Pfleger, J. Mater. Chem. C, 2018, 6, 2533.

31 N. Jia, G. Tian, S. Qi, J. Chen, X. Wang and D. Wu, RSC Adv., 2017, 7, 23550.

32 Y.-Q. Li, R.-C. Fang, A.-M. zheng, Y.-Y. Chu, H.-H. Xu, S.-J. Ding and Y.-Z. Shen, J. Mater. Chem., 2011, 21, 15643.

33 D. M. Kim, Y.-G. Ko, J. K. Choi, K. Kim, W. Kwon, J. Jung, T.-H. Yoon and M. Ree, Polymer, 2012, 53, 1703.

34 Y. Li, Y. Chu, R. Fang, S. Ding, Y. Wang, Y. Shen and A. Zheng, Polymer, 2012, 53, 229.

35 J. Zhao, L. Peng, Y.-L. Zhu, A.-M. Zheng and Y.-Z. Shen, Polym. Chem., 2016, 7, 1765.

36 J. H. Burroughes, D. D. C. Bradley, A. R. Brown, R. N. Marks, K. Mackay, R. H. Friend, P. L. Burns and A. B. Holmes, Nature, 1990, 347, 539.

37 A. Kraft, A. C. Grimsdale and A. B. Holmes, Angew. Chem., Int. Ed., 1998, 37, 402.

38 A. C. Grimsdale, K. L. Chan, R. E. Martin, P. G. Jokisz and A. B. Holmes, Chem. Rev., 2009, 109, 897.

39 M. Mamada, C. Perez-Bolivar and P. Anzenbacher Jr, Org. Lett., 2011, 13, 4882.
40 E. V. Gromachevskaya, A. V. Finko, A. V. Butin, K. S. Pushkareva, V. D. Strelkov, L. I. Isakova and G. D. Krapivin, Chem. Heterocycl. Compd., 2013, 49, 1331.

41 The Sadtler Handbook of Infrared Spectra, in Sadtler Spectral Handbooks, Bio-Rad Laboratories, Inc., Informatics Division, 1978-2004.

42 H.-C. Wu, C.-L. Liu and W.-C. Chen, Polym. Chem., 2013, 4, 5261.

43 V. P. Gupta, in Principles and Applications of Quantum Chemistry, Academic Press, Elsevier Inc., 2016, ch. 6, p. 195.

44 Z. Liu, L. Wang, J. Chen, F. Wang, X. Ouyang and Y. Cao, J. Polym. Sci., Part A: Polym. Chem., 2007, 45, 756.

45 M. S. Liu, J. Luo and A. K.-Y. Jen, Chem. Mater., 2003, 15, 3496.

46 Y.-F. Chang, B. Fowler, Y.-C. Chen, Y.-T. Chen, Y. Wang, F. Xue and F. Zhou, J. Appl. Phys., 2014, 116, 043709.

47 G. Casula, Y. Busby, A. Franquet, V. Spampinato, L. Houssiau, A. Bonfiglio and P. Cosseddu, Org. Electron., 2019, 64, 209.

48 C. Chen, H. Yen, W. Chen and G. Liou, J. Mater. Chem., 2012, 22, 14085.

49 I. Osaka, M. Saito, H. Mori, T. Koganezawa and K. Takimiya, Adv. Mater., 2012, 24, 425.

50 T. Chu, J. Lu, S. Beaupre, Y. Zhang, J. Pouliot, J. Zhou, A. Najari, M. Leclerc and Y. Tao, Adv. Funct. Mater., 2012, 22, 2345.

51 H.-C. Wu, A.-D. Yu, W.-Y. Lee, C.-L. Liu and W.-C. Chen, Chem. Commun., 2012, 48, 9135.

52 Y.-Q. Li, R.-C. Fang, A.-M. Zheng, Y.-Y. Chu, X. Tao, H.-H. Xu, S.-J. Ding and Y.-Z. Shen, J. Mater. Chem., 2011, 21, 15643.

53 D. S. Shang, Q. Wang, D. Chen, R. Dong, X. M. Li and W. Q. Zhang, Phys. Rev. B: Condens. Matter Mater. Phys., 2006, 73, 245427. 